\title{
Generalized universality of higher transverse moments of quark transverse momentum dependent correlators
}

\author{
M.G.A. Buffing, , , 田 A. Mukherjee, $, 2,3$, 团 and P.J. Mulders ${ }^{1, \text { }}$, \\ ${ }^{1}$ Nikhef and Department of Physics and Astronomy, VU University Amsterdam, \\ De Boelelaan 1081, NL-1081 HV Amsterdam, the Netherlands \\ ${ }^{2}$ Department of Physics, Indian Institute of Technology Bombay, Powai, Mumbai 400076, India \\ ${ }^{3}$ Institut für Theoretische Physik, Universität Tübingen, 72076 Tübingen, Germany
}

(Dated: August 28, 2018)

\begin{abstract}
The color gauge-invariant transverse momentum dependent (TMD) quark correlators contain process dependent gauge links in the bilocal matrix elements. In this paper, we split these process dependent correlators into universal TMD correlators, which in turn can be parametrized in universal TMD distribution functions. The process dependence is contained in gluonic pole factors, of which the value is determined by the gauge link. The operator structures of the universal TMD correlators are identified using transverse moments. In this paper, specific results for double transverse weighting of quark TMDs are given. In particular, we show that for a spin $1 / 2$ target one has three universal time-reversal even leading 'pretzelocity distributions', two of which involve double gluonic pole matrix elements and come with process dependent gluonic pole factors. We generalize the results for single and double weighting to TMD correlators of any specific rank, illustrating it for unpolarized, spin $1 / 2$ and spin 1 targets.

PACS numbers: 12.38.-t, 13.85.Ni, 13.85.Qk
\end{abstract}

\section{INTRODUCTION}

The transverse momentum dependent (TMD) correlators for quarks and gluons include not only the dependence on the longitudinal momentum fraction $x$ but also the dependence on the transverse momentum $p_{T}$ of the partons (quarks or gluons). This enables one to incorporate spin-momentum correlations. The correlators in turn are parametrized in terms of parton distribution functions (PDF) and parton fragmentation functions (PFF). The leading TMD distribution and fragmentation functions in these correlators include besides the well-known spin-spin densities that survive in the collinear case (where the transverse momentum is integrated) also spin-orbit densities. These provide for instance a natural interpretation for single spin asymmetries observed at high energies. The correlators are the nonperturbative objects that enter the description of high-energy scattering processes through a convolution with the perturbative hard scattering process. They constitute nonlocal matrix elements of the parton field operators. Collinear gluons exchanged between the soft and hard parts are resummed into the nonperturbative objects and show up as the Wilson lines or color gauge links that make the correlators gauge-invariant. For TMD correlators the nonlocality in the operators is in the transverse direction as well as longitudinal (light-like) direction, and there is no unique way to connect the fields through the gauge link [1-4]. The link depends on the process under consideration. In fact, in the case of single spin asymmetries it is the closing of the gauge link with the transverse gauge link at light-cone plus or minus infinity that plays a major role in distinguishing time-reversal even (T-even) and time-reversal odd (T-odd) TMD distribution and fragmentation functions [5-7]. To study the $p_{T}$-dependence it is convenient to look at the transverse moments, obtained by weighting the TMD functions with one or more powers of $p_{T}$ [2, 8]. It has been shown in Ref. 2] that single weighted correlators relevant for different azimuthal asymmetries can be expressed in terms of two collinear correlators, the first one containing a T-even operator combination and the second one containing a T-odd combination. The latter involves a quark-quark-gluon matrix element with vanishing gluon momentum and is known as the Efremov-Teryaev-Qiu-Sterman (ETQS) or gluonic pole matrix element [9 14]. This matrix element appears in cross sections multiplied with a process dependent gluonic pole factor, which depends on the hard part of the process. Examples of such process dependent T-odd functions are the Sivers and Boer-Mulders function. The $p_{T}$-weighted moment of the fragmentation correlators can also be divided into two parts similar to distributions, but here T-odd effects can come also from the fact that one has complex non-plane wave final states [2]. For fragmentation

\footnotetext{
*Electronic address: m.g.a.buffing@vu.nl

${ }^{\dagger}$ Electronic address: asmita@phy.iitb.ac.in

‡Electronic address: mulders@few.vu.nl
} 
the gluonic pole matrix elements vanish and since for a given transverse moment there is only one specific operator combination, there is no process dependence [15-19]. This is for example the situation for the Collins fragmentation function. Generally, the dependence on the gauge link complicates the universality properties of TMDs as well as factorization issues.

In terms of transverse moments, the study of TMD correlators becomes simpler. There remains a process dependence, but this is dealt with by process dependent gluonic pole factors, that depend on the hard part of the process. In other words, given a process one already knows which correlators are important. While single weighted moments are important for $\cos (\varphi)$ and $\sin (\varphi)$ asymmetries, one needs higher $p_{T}$-moments for $\cos (n \varphi)$ or $\sin (n \varphi)$ asymmetries. For double weighted transverse moments, one looks at weighting with $p_{T}^{\alpha} p_{T}^{\beta}$. The pretzelocity TMD PDF $h_{1 T}^{\perp}\left(x, p_{T}^{2}\right)$ is an example for which double weighting is important. It has received some attention in the literature recently. It is a twist two chiral odd and T-even TMD distribution function. It contributes to the $\sin \left(3 \varphi-\varphi_{S}\right)$ asymmetry in semi-inclusive deep inelastic scattering (SIDIS) 20, 21] and to the $\cos \left(2 \varphi+\varphi_{a}-\varphi_{b}\right)$ asymmetry in the Drell-Yan process involving two transversely polarized protons [22, 23]. In some models, such as the bag model and spectator model, the pretzelocity distribution is shown to be related to the difference between the helicity distribution and the transversity distribution of the nucleon [21]. This relation is not expected to hold in the presence of gluonic interactions. In this work, we analyze the double $p_{T}$-moment of quark correlators taking into account the gauge link, and show that like the first moment these can also be separated into a T-even and a T-odd part. The T-even part contains three contributions, two of them coming from quark-quark-gluon-gluon matrix elements containing two zero momentum gluons, which are double gluonic pole matrix elements. The coefficients of these matrix elements depend on the gauge link $U$ and are process dependent, showing that also the T-even pretzelocity $\operatorname{PDF} h_{1 T}^{\perp[U]}\left(x, p_{T}^{2}\right)$ is nonuniversal. We will show that the pretzelocity function is a combination of universal functions, linked to the three possible T-even matrix elements. While these three functions themselves are by construction universal, it is a particular combination that appears in a given process with link dependence in the multiplicative coefficients. The appearance of three pretzelocity functions is a striking example of how the separation of the correlator into T-even and T-odd contributions is no longer enough to isolate the process dependent part of the correlator when higher transverse moments are involved. In addition to this, we will extend the transverse moment analysis to give definitions of universal $p_{T}^{2}$-dependent functions of a definite rank. This will be done in general for targets with spin and illustrated for unpolarized, spin $1 / 2$ and spin 1 targets.

\section{FORMALISM}

\section{A. Starting points}

The quark-quark TMD correlator is given by

$$
\Phi_{i j}^{[U]}\left(x, p_{T} ; n\right)=\left.\int \frac{d \xi \cdot P d^{2} \xi_{T}}{(2 \pi)^{3}} e^{i p \cdot \xi}\left\langle P\left|\bar{\psi}_{j}(0) U_{[0, \xi]} \psi_{i}(\xi)\right| P\right\rangle\right|_{\xi \cdot n=0}
$$

where we use the Sudakov decomposition $p^{\mu}=x P^{\mu}+p_{T}^{\mu}+\sigma n^{\mu}$ for the momentum $p^{\mu}$ of the produced quark. In this decomposition, $P^{\mu}$ is the momentum of the incoming hadron, which is in essence the leading light-like direction, while $n$ is the conjugate light-like direction satisfying $P \cdot n=0$. The component $\sigma \propto p \cdot P$ along this direction is integrated over. The nonlocal matrix element $\Phi_{i j}^{[U]}\left(x, p_{T} ; n\right)$ contains a process dependent gauge link $U_{[0, \xi]}$, connecting the two fields. The process dependence is in the path of the gauge link. For the two simplest possibilities, the [+] and [-] gauge links, the gauge link runs from 0 to $\xi$ through plus or minus infinity along $n$, respectively. This is illustrated in Fig. 1. More complicated gauge links can arise as well. We refer to Ref. [4] for a detailed description of these gauge

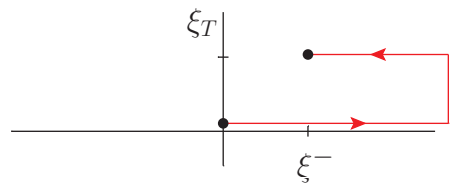

(a)

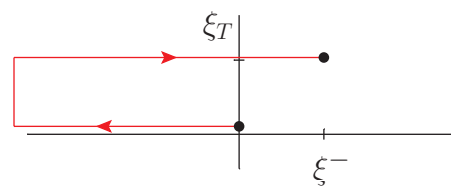

(b)

FIG. 1: The gauge links (a) $[+]$ and (b) $[-]$ running from 0 to $\xi$ with $\xi \cdot n=0$. The light-like separation $\xi^{-}=\xi \cdot P$ and the transverse separation $\xi_{T}$ are nonzero. 
links. After integration over transverse momenta, one has the quark-quark collinear correlator,

$$
\Phi_{i j}^{[U]}(x)=\left.\int \frac{d \xi \cdot P}{2 \pi} e^{i p \cdot \xi}\left\langle P\left|\bar{\psi}_{j}(0) U_{[0, \xi]}^{[n]} \psi_{i}(\xi)\right| P\right\rangle\right|_{\xi \cdot n=0, \xi_{T}=0}
$$

where the gauge link is reduced to a straight-line gauge link or Wilson line, which runs from 0 to $\xi$ along $n$. Since the quark-quark correlators cannot be calculated directly, it is common to make a parametrization that contains TMD or collinear PDFs, respectively. In the TMD case, there are for a spin 1/2 nucleon eight leading contributing terms in the parametrization of the TMD correlator [24],

$$
\begin{aligned}
\Phi^{[U]}\left(x, p_{T} ; n\right)= & \left\{f_{1}^{[U]}\left(x, p_{T}^{2}\right)-f_{1 T}^{\perp[U]}\left(x, p_{T}^{2}\right) \frac{\epsilon_{T}^{\rho \sigma} p_{T \rho} S_{T \sigma}}{M}+g_{1 s}^{[U]}\left(x, p_{T}\right) \gamma_{5}\right. \\
& \left.+h_{1 T}^{[U]}\left(x, p_{T}^{2}\right) \gamma_{5} \$_{T}+h_{1 s}^{\perp[U]}\left(x, p_{T}\right) \frac{\gamma_{5} \not p_{T}}{M}+i h_{1}^{\perp[U]}\left(x, p_{T}^{2}\right) \frac{\not p_{T}}{M}\right\} \frac{\not P}{2},
\end{aligned}
$$

with the spin vector parametrized as $S^{\mu}=S_{L} P^{\mu}+S_{T}^{\mu}+M^{2} S_{L} n^{\mu}$ and shorthand notations for $g_{1 s}^{[U]}$ and $h_{1 s}^{\perp[U]}$,

$$
g_{1 s}^{[U]}\left(x, p_{T}\right)=S_{L} g_{1 L}^{[U]}\left(x, p_{T}^{2}\right)-\frac{p_{T} \cdot S_{T}}{M} g_{1 T}^{[U]}\left(x, p_{T}^{2}\right)
$$

The TMD distribution functions in this parametrization depend on $x$ and $p_{T}^{2}=-\boldsymbol{p}_{T}^{2}=-\left|p_{T}\right|^{2}$. The leading contributions in the correlator all have a $\not P$ factor and are distinguished by different azimuthal behavior for the transverse vectors such as $p_{T}$ and $S_{T}$. The correlators and the TMD distribution functions in the parametrization also depend on the gauge link. Time-reversal relates the functions in $\Phi^{[U]}$ to those in $\Phi^{\left[U^{t}\right]}$, where $U^{t}$ is the time-reversed gauge link, which means interchanging the running via light-cone plus or minus infinity. For the functions $f_{1 T}^{\perp}$ and $h_{1}^{\perp}$ one has $f_{1 T}^{\perp[U]}=-f_{1 T}^{\perp\left[U^{t}\right]}$, a property that is referred to as naive T-odd. Each TMD has either zero, one or two factors of $p_{T}$ as a prefactor. This will play a role when integrations over transverse momenta are considered. It is actually useful to use in the parametrization irreducible (symmetric and traceless) tensors in the transverse space,

$$
p_{T}^{\alpha}, \quad p_{T}^{\alpha \beta}=p_{T}^{\alpha} p_{T}^{\beta}-\frac{1}{2} p_{T}^{2} g_{T}^{\alpha \beta}, \ldots
$$

Just integrating (without weights) Eq. 3 over transverse momenta, only the contributions without prefactor of $p_{T}$ or traces survive, yielding

$$
\Phi(x)=\left\{f_{1}(x)+S_{L} g_{1}(x) \gamma_{5}+h_{1}(x) \gamma_{5} \$_{T}\right\} \frac{\not p}{2}
$$

at the leading twist two level. Here $g_{1}(x)$ is the integrated version of $g_{1 L}^{[U]}\left(x, p_{T}^{2}\right)$ and $h_{1}(x)$ is the $p_{T}$-integrated version of $h_{1}^{[U]}\left(x, p_{T}^{2}\right)=h_{1 T}^{[U]}\left(x, p_{T}^{2}\right)+h_{1 T}^{\perp[U](1)}\left(x, p_{T}^{2}\right)$ including a trace term, which involves functions weighted with powers of $-p_{T}^{2} / 2 M^{2}=\boldsymbol{p}_{T}^{2} / 2 M^{2}$, in general

$$
f_{\ldots}^{(n)}\left(x, p_{T}^{2}\right)=\left(\frac{-p_{T}^{2}}{2 M^{2}}\right)^{n} f_{\ldots}\left(x, p_{T}^{2}\right) .
$$

The integrated functions $f_{\ldots}^{(n)}(x)$ are usually referred to as transverse moments, but we will extend this name to azimuthally averaged functions that still depend on $p_{T}^{2}$. The collinear PDFs in Eq. 6 are independent of the gauge link $U$. In other words, all operator definitions of these collinear PDFs have a unique straight-line gauge link.

The behavior of (TMD) PDFs under time-reversal can be studied. The functions $f_{1 T}^{\perp}$ and $h_{1}^{\perp}$ are time-reversal odd (T-odd), while the remaining six functions are time-reversal even (T-even). Similarly, one can look at the behavior of the matrix element(s) under time-reversal. Using the fact that the simplest gauge links for quark correlators, the $[+]$ and [-] gauge links, are a time-reversal couple, one can construct T-even and T-odd TMD correlators [2],

$$
\begin{aligned}
& \Phi^{\text {(T-even) }}\left(x, p_{T}\right)=\frac{1}{2}\left(\Phi^{[+]}\left(x, p_{T}\right)+\Phi^{[-]}\left(x, p_{T}\right)\right) \\
& \Phi^{\text {(T-odd })}\left(x, p_{T}\right)=\frac{1}{2}\left(\Phi^{[+]}\left(x, p_{T}\right)-\Phi^{[-]}\left(x, p_{T}\right)\right) .
\end{aligned}
$$

For the unweighted integrated case the separation between T-even and T-odd objects would be trivial, since the $[+]$ and [-] gauge links are identical after integration over transverse momentum. As a result, $\Phi(x)=\Phi^{(\mathrm{T}-\mathrm{even})}(x)$ and $\Phi^{\text {(T-odd) }}(x)=0$. For the transverse momentum weighted case both functions are important. One thus is tempted to identify the TMD functions $f_{1 T}^{\perp}$ and $h_{1}^{\perp}$ to the T-odd correlator and the other TMD functions to the T-even correlator, in which the T-odd ones acquire process dependence. The situation will turn out to be more complex, which is most easily demonstrated by looking at transverse momentum weighting. 


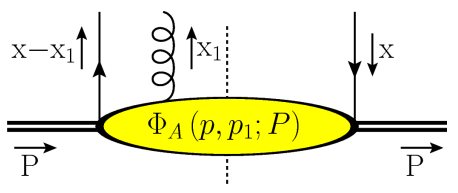

(a)

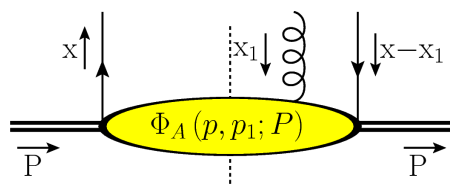

(b)

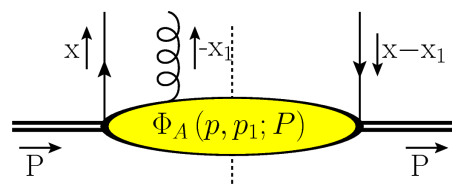

(c)

FIG. 2: The correlators (a) $\Phi_{A}\left(x-x_{1}, x_{1} \mid x\right)$, (b) $\Phi_{A}\left(x \mid x_{1}, x-x_{1}\right)$ and (c) $\Phi_{A}\left(x,-x_{1} \mid x-x_{1}\right)$. Note that the diagrams in (b) and (c) are equal to each other.

\section{B. Single transverse weighting}

In the $p_{T}$-weighted case multiple matrix elements appear, since the transverse weighting gives rise to a derivative that not only acts on the fields, but on the gauge links as well. Weighting with $p_{T}^{\alpha}$ can be rewritten in terms of two contributions, which upon $p_{T}$-integration only depend on $x$ and depend on the link just through a gluonic pole factor [2],

$$
\begin{aligned}
\Phi_{\partial}^{\alpha[U]}(x) \equiv \int d^{2} p_{T} p_{T}^{\alpha} \Phi^{[U]}\left(x, p_{T}\right) & =\left(\Phi_{D}^{\alpha}(x)-\Phi_{A}^{\alpha}(x)\right)+\pi C_{G}^{[U]} \Phi_{G}^{\alpha}(x) \\
& =\widetilde{\Phi}_{\partial}^{\alpha}(x)+\pi C_{G}^{[U]} \Phi_{G}^{\alpha}(x) .
\end{aligned}
$$

The matrix element $\Phi_{G}^{\alpha}(x)$ is referred to as the gluonic pole matrix element. All matrix elements are built from multiparton twist three operator combinations, illustrated in Fig. 2. The relevant ones in the final result of a calculation involve gauge-invariant operators $i D_{T}^{\alpha}$ and $F_{T}^{n \alpha}$ rather than $A_{T}^{\alpha}$,

$$
\begin{aligned}
& \Phi_{D i j}^{\alpha}\left(x-x_{1}, x_{1} \mid x\right)=\left.\int \frac{d \xi \cdot P d \eta \cdot P}{(2 \pi)^{2}} e^{i p_{1} \cdot \eta+i\left(p-p_{1}\right) \cdot \xi}\left\langle P\left|\bar{\psi}_{j}(0) U_{[0, \eta]} i D_{T}^{\alpha}(\eta) U_{[\eta, \xi]} \psi_{i}(\xi)\right| P\right\rangle\right|_{L C}, \\
& \Phi_{F i j}^{\alpha}\left(x-x_{1}, x_{1} \mid x\right)=\left.\int \frac{d \xi \cdot P d \eta \cdot P}{(2 \pi)^{2}} e^{i p_{1} \cdot \eta+i\left(p-p_{1}\right) \cdot \xi}\left\langle P\left|\bar{\psi}_{j}(0) U_{[0, \eta]} F_{T}^{n \alpha}(\eta) U_{[\eta, \xi]} \psi_{i}(\xi)\right| P\right\rangle\right|_{L C} .
\end{aligned}
$$

The matrix elements showing up in Eq. 9 are related to these multi-parton correlators, to be precise we need the matrix elements

$$
\begin{aligned}
\Phi_{D}^{\alpha}(x) & =\int d x_{1} \Phi_{D}^{\alpha}\left(x-x_{1}, x_{1} \mid x\right), \\
\Phi_{A}^{\alpha}(x) & \equiv \int d x_{1} \operatorname{PV} \frac{i}{x_{1}} \Phi_{F}^{n \alpha}\left(x-x_{1}, x_{1} \mid x\right), \\
\Phi_{G}^{\alpha}(x) & =\Phi_{F}^{n \alpha}(x, 0 \mid x) .
\end{aligned}
$$

By using the Eqs $8 \mathrm{a}$ and $8 \mathrm{~b}$, one finds that $\widetilde{\Phi}_{\partial}^{\alpha}(x)$ is a T-even matrix element, involving the $\Phi_{D}$ and $\Phi_{A}$, the latter being the principal value integration over a correlator involving the gluon fields defined in a T-invariant way. The gluonic pole matrix element $\Phi_{G}^{\alpha}(x)$, in order to distinct it from $\Phi_{F}$ indicated with an index $G$, is T-odd.

The choice of notation for the arguments of multiparton correlators, where the produced quark and gluon on the left side of the cut have momentum fractions $x-x_{1}$ and $x_{1}$ and the incoming quark on the right side of the cut has a momentum fraction $x$, is illustrated in Fig. 2a. Despite the fact that the assignment of momenta in the correlators in Eqs 10,14 is overdetermined, it has the advantage that it is more transparent in our forthcoming generalization to higher weightings. Furthermore, because of the absence of T-ordering, one can move a gluon through the cut by changing the sign of the momentum. Under hermiticity one finds that the correlators in Eq. 14 have the behavior $\gamma_{0} \Phi_{A}^{\dagger}\left(x-x_{1}, x_{1} \mid x\right) \gamma_{0}=\Phi_{A}\left(x \mid x_{1}, x-x_{1}\right)$.

The weighting with transverse momenta can also be analyzed by studying the parametrization in PDFs. For single $p_{T}$-weighting, only PDFs with one prefactor of $p_{T}$ in the parametrization in Eq. 3 survive. T-even PDFs contribute to the $\widetilde{\Phi}_{\partial}^{\alpha}(x)$ matrix element, while T-odd PDFs contribute to the $\Phi_{G}^{\alpha}(x)$ matrix element, see Ref. [2] for a detailed study of this. Since the T-odd matrix element comes with a process dependent prefactor, it can be seen that for single $p_{T}$-weighting, the behavior under time-reversal can be used to identify the process dependent parts. In literature, it

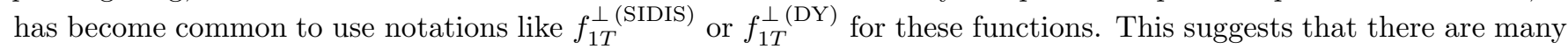
different versions of specific functions, which are obviously not universal, one for each process with a different gauge 


\begin{tabular}{r|c|c|c|}
\multicolumn{1}{l|}{$U$} & $U^{[ \pm]}$ & $U^{[+]} U^{[\square]}$ & $\frac{1}{N_{c}} \operatorname{Tr}_{c}\left(U^{[\square]}\right) U^{[+]}$ \\
\hline$\Phi^{[U]}$ & $\Phi^{[ \pm]}$ & $\Phi^{[+\square]}$ & $\Phi^{[(\square)+]}$ \\
\hline$C_{G}^{[U]}$ & \pm 1 & 3 & 1 \\
\hline$C_{G G, 1}^{[U]}$ & 1 & 9 & 1 \\
\hline$C_{G G, 2}^{[U]}$ & 0 & 0 & 4 \\
\hline
\end{tabular}

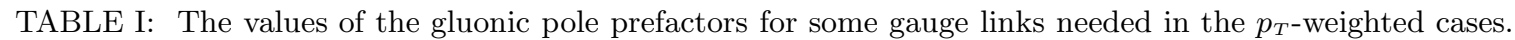
Note that the value of $C_{G}^{[U]}$ is the same for single and double transverse weighting.

link. In fact there is a universal transverse moment relating all link dependent ones

$$
f_{1 T}^{\perp(1)[U]}(x)=C_{G}^{[U]} f_{1 T}^{\perp(1)}(x) .
$$

Although the only difference for the single weighted case is just the numerical prefactor that for simple processes is just +1 or -1 , we will show in the next section that for the double weighted case the situation becomes more complicated and one actually gains a lot by this different notation. But even for single weighting there is a clear advantage using Eq. 15, because it states that there is a universal function with calculable process (link) dependent numbers rather than an infinite number of somehow related functions. For some gauge links, these numbers are shown in Table I. Here $U^{[\square]}$ is the Wilson loop $U^{[-]^{\dagger}} U^{[+]}$.

\section{Double transverse weighting}

In order to evaluate the double transverse weighting we need to consider matrix elements like

$$
\begin{aligned}
\Phi_{F F}^{\alpha \beta}\left(x-x_{1}-x_{2}, x_{1}, x_{2} \mid x\right)= & \int \frac{d \xi \cdot P}{2 \pi} \frac{d \eta \cdot P}{2 \pi} \frac{d \eta^{\prime} \cdot P}{2 \pi} e^{i x_{2}\left(\eta^{\prime} \cdot P\right)} e^{i x_{1}(\eta \cdot P)} e^{i\left(x-x_{1}-x_{2}\right)(\xi \cdot P)} \\
& \times\left.\left\langle P, S\left|\bar{\psi}(0) U_{\left[0, \eta^{\prime}\right]}^{[n]} F_{T}^{n \alpha}\left(\eta^{\prime}\right) U_{\left[\eta^{\prime}, \eta\right]}^{[n]} F_{T}^{n \beta}(\eta) U_{[\eta, \xi]}^{[n]} \psi(\xi)\right| P, S\right\rangle\right|_{L C},
\end{aligned}
$$

among others, where $L C$ indicates that all transverse components and $n$-components of the coordinates are zero. Besides this matrix element one needs $\Phi_{D F}, \Phi_{F D}$ and $\Phi_{D D}$ as well as bilocal matrix elements, obtained by direct or principal value integrations over these matrix elements (as in the case of single transverse momentum weighting) or gluonic pole matrix elements, where $x_{1}$ or $x_{2}$ or both are zero. Explicitly, the matrix elements are discussed in Appendix $\mathrm{A}$.

The actual weighting of the gauge link dependent TMD correlator $\Phi^{[U]}\left(x, p_{T}\right)$ gives

$$
\begin{aligned}
\Phi_{\partial \partial}^{\{\alpha \beta\}[U]}(x) & \equiv \int d^{2} p_{T} p_{T}^{\{\alpha} p_{T}^{\beta\}} \Phi^{[U]}\left(x, p_{T}^{2}\right) \\
& =\widetilde{\Phi}_{\partial \partial}^{\{\alpha \beta\}}(x)+\pi C_{G}^{[U]}\left(\widetilde{\Phi}_{\partial G}^{\{\alpha \beta\}}(x)+\widetilde{\Phi}_{G \partial}^{\{\alpha \beta\}}(x)\right)+\sum_{c} \pi^{2} C_{G G, c}^{[U]} \Phi_{G G, c}^{\{\alpha \beta\}}(x) \\
& =\widetilde{\Phi}_{\partial \partial}^{\{\alpha \beta\}}(x)+\pi C_{G}^{[U]}\left(\widetilde{\Phi}_{\partial G}^{\{\alpha \beta\}}(x)+\widetilde{\Phi}_{G \partial}^{\{\alpha \beta\}}(x)\right)+\pi^{2} C_{G G, 1}^{[U]} \Phi_{G G, 1}^{\{\alpha \beta\}}(x)+\pi^{2} C_{G G, 2}^{[U]} \Phi_{G G, 2}^{\{\alpha \beta\}}(x) .
\end{aligned}
$$

For the correlators containing two (or more) gluon fields like the one in Eq. 16, one must distinguish the different color structures for the correlator, hence a summation over the color structures $c$. For double weighting, there are in the double gluonic pole part two possible color structures related to the appearance of the color traced Wilson loop $\frac{1}{N_{c}} \operatorname{Tr}_{c}\left(U^{[\square]}\right)$. The differences between the two different correlators $\Phi_{G G, c}^{\{\alpha \beta\}}(x)$ are made explicit in Appendix $\mathrm{A}$, Just as for the single weighted case in Eq. 9, the structures $\widetilde{\Phi}_{\ldots}$ with one or more partial derivatives denote differences between correlators with a covariant derivative minus a correlator with a principal value integration, e.g. $\widetilde{\Phi}_{\partial G}^{\{\alpha \beta\}}(x)=$ $\Phi_{D G}^{\{\alpha \beta\}}(x)-\Phi_{A G}^{\{\alpha \beta\}}(x)$. For completeness, they are given in Appendix $\mathrm{A}$ Since the weighting is done with the symmetric combination, we have symmetrized in the indices, which should not influence the result. We also omitted the Dirac indices on the fields. The precise form of all correlators in terms of matrix elements can be found in Appendix $\mathrm{A}$, 
The only leading twist TMD PDF that contributes is the pretzelocity $h_{1 T}^{\perp[U]}\left(x, p_{T}^{2}\right)$. Since this function is T-even, its (double) transverse moment could be associated with both the matrix elements $\widetilde{\Phi}_{\partial \partial}^{\{\alpha \beta\}}(x)$ and $\pi^{2} \Phi_{G G, c}^{\{\alpha \beta\}}(x)$. The gluonic pole matrix elements come with gauge link dependent prefactors, so the pretzelocity function as it has been defined in literature up to now is not universal. The double gluonic pole factor gives the gauge link dependence and one must identify the gauge link dependent function as the sum of three functions,

$$
h_{1 T}^{\perp(2)[U]}(x)=h_{1 T}^{\perp(2)(A)}(x)+C_{G G, 1}^{[U]} h_{1 T}^{\perp(2)(B 1)}(x)+C_{G G, 2}^{[U]} h_{1 T}^{\perp(2)(B 2)}(x),
$$

where the functions $h_{1 T}^{\perp(2)(A)}(x), h_{1 T}^{\perp(2)(B 1)}(x)$ and $h_{1 T}^{\perp(2)(B 2)}(x)$ are universal. For simple processes like SIDIS and Drell-Yan with $C_{G G, 1}=1$ and $C_{G G, 2}=0$ one finds just the sum of two pretzelocity functions. For processes with a more complicated gauge link structure, other combinations involving three functions will appear, as can be seen in Table [. The double-weighted results also show that for higher transverse moments, and hence also for the full $p_{T}$-dependent treatment, a separation in T-odd and T-even functions is no longer sufficient to isolate the process dependent parts.

\section{DEFINING TMDS}

For the definition of a TMD correlator parametrized in terms of PDFs (or PFFs) depending on the collinear fraction $x$ and transverse momentum $p_{T}$ it is important to keep in mind the role of $x$ and $p_{T}$. These are identified with kinematic variables in a high-energy scattering process. This is most well-known for $x$, which in a SIDIS process is identified with the Bjorken scaling variable. In the same way the transverse momentum can be identified, e.g. from the noncollinearity of produced hadrons or from jet-jet asymmetries, even if for transverse momenta the identification is usually contained in a folding of transverse momenta of several hadron correlators. So for purposes of further analyzing we assume that we know that a hadron correlator depends on 'measurable' $x$ and $p_{T}$. When including the additional collinear gluons producing the gauge link, $p_{T}$ is the sum of all transverse momenta of the partons exchanged between the (soft) hadron correlator and the hard process. This requires integration over the transverse momenta of collinear gluons.

The leading relevant TMD operator structure for our considerations thus will be of the generic bilocal form

$$
\Phi^{[U]}\left(x, p_{T} ; n\right)=\left.\int \frac{d \xi \cdot P d^{2} \xi_{T}}{(2 \pi)^{3}} e^{i p \cdot \xi}\left\langle P\left|\bar{\psi}(0) U_{[0, \xi]} O(\xi) \psi(\xi)\right| P\right\rangle\right|_{\xi \cdot n=0},
$$

where $U$ is one of the possible gauge links for TMD correlators. We can define in this way $\widetilde{\Phi}_{\partial}^{\alpha}\left(x, p_{T}\right)=\Phi_{D}^{\alpha}\left(x, p_{T}\right)-$ $\Phi_{A}^{\alpha}\left(x, p_{T}\right)$, as well as correlators $\Phi_{D D}^{\alpha \beta}, \Phi_{D A}^{\alpha \beta}, \ldots, \widetilde{\Phi}_{\partial \partial}^{\alpha \beta}, \widetilde{\Phi}_{\partial G}^{\alpha \beta}, \ldots, \Phi_{G G}^{\alpha \beta}$, where one in particular for $\Phi_{G G}$ must take care of the color structure.

Our identification of operator structures and TMD functions in the parametrization of correlators depends on the comparison of moments in $x$ and $p_{T}$, even if such moments in real life are limited by kinematics of the process. This is well-known, but nontrivial, for the collinear dependence, where the moments can be related to local matrix elements of quark and gluon fields. All these operators have the same twist (canonical dimension minus rank of Lorentz indices), which means they contribute at the same order of the hard scale. The $x^{N-1}$ Mellin moments correspond to expectation values of leading twist operators of rank $N$. These local matrix elements have a calculable scale dependence governed by the anomalous dimension of the local operator. The scale is usually identified with the kinematic limit such as the exchanged momentum $Q^{2}$ in SIDIS. The $x$-dependent functions can be reconstructed from the Mellin moments. Their scale dependence then is obtained by folding them with splitting functions, of which the Mellin moments are precisely the anomalous dimensions.

\section{A. TMDs of definite rank}

For the $p_{T}$-dependent functions we follow a similar procedure. An expansion of TMDs involves the symmetric traceless tensors $p_{T}^{\alpha_{1} \ldots \alpha_{m}}$ of rank $m$. These traceless tensors satisfy

$$
\int d^{2} p_{T} p_{T}^{\alpha_{1} \ldots \alpha_{m}} p_{T i_{1} \ldots i_{m}} f \ldots\left(x, p_{T}^{2}\right) \propto f_{\ldots}^{(m)}(x) .
$$

Actually, it is sufficient and for our purposes desirable to integrate only the azimuthal part,

$$
\int \frac{d \varphi_{p}}{2 \pi} p_{T}^{\alpha_{1} \ldots \alpha_{m}} p_{T i_{1} \ldots i_{m}} f_{\ldots}\left(x, p_{T}^{2}\right) \propto f_{\ldots}^{(m)}\left(x, p_{T}^{2}\right) .
$$


The r.h.s. of these equations contain the transverse moments $f^{(m)}$ defined in Eq. 7 as well as constant tensors without azimuthal dependence. Writing the following parametrization,

$$
\begin{aligned}
\Phi^{[U]}\left(x, p_{T}\right)= & \Phi\left(x, p_{T}^{2}\right)+\pi C_{G}^{[U]} \frac{p_{T i}}{M} \Phi_{G}^{i}\left(x, p_{T}^{2}\right)+\pi^{2} C_{G G, c}^{[U]} \frac{p_{T i j}}{M^{2}} \Phi_{G G, c}^{i j}\left(x, p_{T}^{2}\right)+\pi^{3} C_{G G G, c}^{[U]} \frac{p_{T i j k}}{M^{3}} \Phi_{G G G, c}^{i j k}\left(x, p_{T}^{2}\right)+\ldots \\
& +\frac{p_{T i}}{M} \widetilde{\Phi}_{\partial}^{i}\left(x, p_{T}^{2}\right)+\pi C_{G}^{[U]} \frac{p_{T i j}}{M^{2}} \widetilde{\Phi}_{\{\partial G\}}^{i j}\left(x, p_{T}^{2}\right)+\pi^{2} C_{G G, c}^{[U]} \frac{p_{T i j k}}{M^{3}} \widetilde{\Phi}_{\{\partial G G\}, c}^{i j k}\left(x, p_{T}^{2}\right)+\ldots \\
& +\frac{p_{T i j}}{M^{2}} \widetilde{\Phi}_{\partial \partial}^{i j}\left(x, p_{T}^{2}\right)+\pi C_{G}^{[U]} \frac{p_{T i j k}}{M^{3}} \widetilde{\Phi}_{\{\partial \partial G\}}^{i j k}\left(x, p_{T}^{2}\right)+\ldots \\
& +\frac{p_{T i j k}}{M^{3}} \widetilde{\Phi}_{\partial \partial \partial}^{i j k}\left(x, p_{T}^{2}\right)+\ldots,
\end{aligned}
$$

we reproduce the moments. Note that in Eq. 22 depending on the gauge link there are multiple contributing color structures for terms with two or more gluonic pole terms, hence the inclusion of the summation over these color structures $c$. For the collinear correlators $\Phi_{G G, c}^{i j}(x)$ this is discussed in Appendix $\mathrm{A}$ for the TMD correlator $\Phi_{G G, c}^{i j}\left(x, p_{T}^{2}\right)$ it is discussed in Appendix B. The operator structures on the r.h.s. thus are the ones appearing in an angular expansion, in which the azimuthal dependence is made explicit. The combinations like $\widetilde{\Phi}_{\{\partial G\}}$ indicate symmetrized combinations $\widetilde{\Phi}_{\{\partial G\}}=\widetilde{\Phi}_{\partial G}+\widetilde{\Phi}_{G \partial}$. Upon $\varphi$-integration only the structure $\Phi\left(x, p_{T}^{2}\right)$ survives. Hence, we identify this as the rank zero TMD correlator,

$$
\Phi\left(x, p_{T}^{2}\right)=\left\{f_{1}\left(x, p_{T}^{2}\right)+S_{L} g_{1}\left(x, p_{T}^{2}\right) \gamma_{5}+h_{1}\left(x, p_{T}^{2}\right) \gamma_{5} \$_{T}\right\} \frac{\not P}{2}
$$

where the TMD correlator $h_{1}\left(x, p_{T}^{2}\right)$ rather than $h_{1 T}\left(x, p_{T}^{2}\right)$ appears (see also the remark following Eq. 6). Next, we look at the weighted expressions before $p_{T}$-integration in order to explicitly identify further TMD functions,

$$
\begin{aligned}
\frac{p_{T}^{\alpha}}{M} \Phi^{[U]}\left(x, p_{T}\right)= & \frac{p_{T}^{\alpha}}{M} \Phi\left(x, p_{T}^{2}\right)-\widetilde{\Phi}_{\partial}^{\alpha(1)}\left(x, p_{T}^{2}\right)-\pi C_{G}^{[U]} \Phi_{G}^{\alpha(1)}\left(x, p_{T}^{2}\right) \\
& +\frac{p_{T}^{\alpha} i}{M^{2}} \widetilde{\Phi}_{\partial}^{i}\left(x, p_{T}^{2}\right)+\pi C_{G}^{[U]} \frac{p_{T}^{\alpha} i}{M^{2}} \Phi_{G}^{i}\left(x, p_{T}^{2}\right)+\ldots
\end{aligned}
$$

The first term is obviously a term that needs to be there because we already identified a nonzero rank 0 TMD correlator. The next two terms involve the T-even and T-odd transverse moments of $\widetilde{\Phi}_{\partial}^{\alpha}\left(x, p_{T}^{2}\right)$ and $\Phi_{G}^{\alpha}\left(x, p_{T}^{2}\right)$, respectively, which survive $\varphi$-integration. The other terms in Eq. 24] contain higher rank tensors in $p_{T}$. Comparing the unintegrated expression with the parametrization for a spin $1 / 2$ target one thus immediately identifies in addition to the rank 0 correlator in Eq. 23 two rank one TMD correlators,

$$
\begin{aligned}
& \frac{p_{T i}}{M} \widetilde{\Phi}_{\partial}^{i}\left(x, p_{T}^{2}\right)=\left\{h_{1 L}^{\perp}\left(x, p_{T}^{2}\right) S_{L} \frac{\gamma_{5} \not p_{T}}{M}-g_{1 T}\left(x, p_{T}^{2}\right) \frac{p_{T} \cdot S_{T}}{M} \gamma_{5}\right\} \frac{\not P}{2}, \\
& \frac{p_{T i}}{M} \Phi_{G}^{i}\left(x, p_{T}^{2}\right)=\frac{1}{\pi}\left\{-f_{1 T}^{\perp}\left(x, p_{T}^{2}\right) \frac{\epsilon_{T}^{\rho \sigma} p_{T \rho} S_{T \sigma}}{M}+i h_{1}^{\perp}\left(x, p_{T}^{2}\right) \frac{\not p_{T}}{M}\right\} \frac{\not P}{2},
\end{aligned}
$$

the first one being T-even, the second one T-odd.

Before going to double weighting, it is useful to realize that in Eq. 22 one does not need to subtract trace terms from the second rank correlators. This is automatic because of the use of the tensor $p_{T}^{\alpha \beta}$. We can write

$$
\frac{p_{T i j}}{M^{2}} \widetilde{\Phi}_{\{\partial G\}}^{i j}=\left(\frac{p_{T i} p_{T j}}{M^{2}}-\frac{p_{T}^{2}}{2 M^{2}} g_{T i j}\right) \widetilde{\Phi}_{\{\partial G\}}^{i j}=\frac{p_{T i} p_{T j}}{M^{2}} \widetilde{\Phi}_{\{\partial G\}}^{i j}+\widetilde{\Phi}_{\{\partial \cdot G\}}^{(1)}=\frac{p_{T i} p_{T j}}{M^{2}}\left(\widetilde{\Phi}_{\{\partial G\}}^{i j}-\frac{1}{2} g_{T}^{i j} \widetilde{\Phi}_{\{\partial \cdot G\}}\right) .
$$

In Eq. 27 we introduced the notation $\partial \cdot G$ in the subscript of one of the correlators to indicate that these two operators in this correlator have been contracted. Also for double $p_{T}$-weighting we write down (selected contributions in) the unintegrated result starting with Eq. 22, We find for the double weighted result,

$$
\begin{aligned}
\frac{p_{T}^{\alpha} p_{T}^{\beta}}{M^{2}} \Phi^{[U]}\left(x, p_{T}\right)= & \frac{p_{T}^{\alpha} p_{T}^{\beta}}{M^{2}} \Phi\left(x, p_{T}^{2}\right)-\frac{1}{2} g_{T}^{\alpha \beta} \frac{p_{T i}}{M} \widetilde{\Phi}_{\partial}^{i(1)}\left(x, p_{T}^{2}\right)-\frac{1}{2} \pi C_{G}^{[U]} g_{T}^{\alpha \beta} \frac{p_{T i}}{M} \Phi_{G}^{i(1)}\left(x, p_{T}^{2}\right) \\
& -\frac{1}{2 M} p_{T}^{\{\alpha} \widetilde{\Phi}_{\partial}^{\beta\}(1)}\left(x, p_{T}^{2}\right)-\pi C_{G}^{[U]} \frac{1}{2 M} p_{T}^{\{\alpha} \Phi_{G}^{\beta\}(1)}\left(x, p_{T}^{2}\right)+\ldots \\
& +\widetilde{\Phi}_{\partial \partial}^{\alpha \beta(2)}\left(x, p_{T}^{2}\right)+\pi C_{G}^{[U]} \widetilde{\Phi}_{\{\partial G\}}^{\alpha \beta(2)}\left(x, p_{T}^{2}\right)+\pi^{2} C_{G G, c}^{[U]} \Phi_{G G, c}^{\alpha \beta(2)}\left(x, p_{T}^{2}\right)+\ldots \\
& -\frac{1}{2} g_{T}^{\alpha \beta}\left(\widetilde{\Phi}_{\partial \cdot \partial}^{(2)}\left(x, p_{T}^{2}\right)+\pi C_{G}^{[U]} \widetilde{\Phi}_{\{\partial \cdot G\}}^{(2)}\left(x, p_{T}^{2}\right)+\pi^{2} C_{G G, c}^{[U]} \Phi_{G \cdot G, c}^{(2)}\left(x, p_{T}^{2}\right)\right)+\ldots
\end{aligned}
$$


an equation that after symmetrizing in $\alpha$ and $\beta$ can be used to identify the remaining TMDs for a spin $1 / 2$ target. We have omitted terms with rank 1 tensors in $p_{T}$ multiplying $\widetilde{\Phi}_{\partial \partial \partial}^{\alpha \beta i}$, etc., as well as terms with rank 3 or rank 4 tensors like $p_{T i}^{\alpha \beta}$ and $p_{T}^{\alpha \beta}{ }_{i j}$. The last line in Eq. 28 containing terms that arise by contraction of the indices $i$ and $j$ in the explicit rewriting of the product $p_{T}^{\alpha} p_{T}^{\beta} p_{T i j}$ through the contribution $g_{T}^{\alpha \beta} g_{T i j}$, is included for completeness. Note that taking the trace $-\frac{1}{2} g_{T \alpha \beta}$ gives

$$
\begin{aligned}
-\frac{p_{T}^{2}}{2 M^{2}} \Phi^{[U]}\left(x, p_{T}\right)= & \Phi^{(1)}\left(x, p_{T}^{2}\right)+\frac{p_{T i}}{M} \widetilde{\Phi}_{\partial}^{i(1)}\left(x, p_{T}^{2}\right)+\pi C_{G}^{[U]} \frac{p_{T i}}{M} \Phi_{G}^{i(1)}\left(x, p_{T}^{2}\right) \\
& -\frac{1}{2} \widetilde{\Phi}_{\partial \cdot \partial}^{(2)}\left(x, p_{T}^{2}\right)-\frac{1}{2} \pi C_{G}^{[U]} \widetilde{\Phi}_{\{\partial \cdot G\}}^{(2)}\left(x, p_{T}^{2}\right)-\frac{1}{2} \pi^{2} C_{G G, c}^{[U]} \Phi_{G \cdot G, c}^{(2)}\left(x, p_{T}^{2}\right)+\ldots \\
& +\left(\widetilde{\Phi}_{\partial \cdot \partial}^{(2)}\left(x, p_{T}^{2}\right)+\pi C_{G}^{[U]} \widetilde{\Phi}_{\{\partial \cdot G\}}^{(2)}\left(x, p_{T}^{2}\right)+\pi^{2} C_{G G, c}^{[U]} \Phi_{G \cdot G, c}^{(2)}\left(x, p_{T}^{2}\right)\right)+\ldots,
\end{aligned}
$$

where the terms between brackets come from the correlators with contracted operators in Eq. 28. This shows that

$$
\begin{aligned}
\frac{p_{T}^{\alpha \beta}}{M^{2}} \Phi^{[U]}\left(x, p_{T}\right)= & \frac{p_{T}^{\alpha \beta}}{M^{2}} \Phi\left(x, p_{T}^{2}\right) \\
& -\frac{1}{2 M}\left(p_{T}^{\{\alpha} \widetilde{\Phi}_{\partial}^{\beta\}(1)}\left(x, p_{T}^{2}\right)-\text { trace }\right)-\frac{1}{2 M} \pi C_{G}^{[U]}\left(p_{T}^{\{\alpha} \Phi_{G}^{\beta\}(1)}\left(x, p_{T}^{2}\right)-\text { trace }\right)+\ldots \\
& +\left(\widetilde{\Phi}_{\partial \partial}^{\alpha \beta(2)}\left(x, p_{T}^{2}\right)-\text { traces }\right)+\pi C_{G}^{[U]}\left(\widetilde{\Phi}_{\{\partial G\}}^{\alpha \beta(2)}\left(x, p_{T}^{2}\right)-\text { traces }\right) \\
& +\pi^{2} C_{G G, c}^{[U]}\left(\Phi_{G G, c}^{\alpha \beta(2)}\left(x, p_{T}^{2}\right)-\operatorname{traces}_{c}\right)+\ldots,
\end{aligned}
$$

illustrating how the projection with the properly symmetrized traceless second rank tensor $p_{T}^{\alpha \beta}$ gives the properly symmetrized traceless TMD structures. The terms without azimuthal dependence are identified with the rank two TMD correlators, which for a spin $1 / 2$ target are parametrized as

$$
\begin{aligned}
& \frac{p_{T i j}}{M^{2}} \widetilde{\Phi}_{\partial \partial}^{i j}\left(x, p_{T}^{2}\right)=h_{1 T}^{\perp(A)}\left(x, p_{T}^{2}\right) \frac{p_{T i j} S_{T}^{i} \gamma_{5} \gamma_{T}^{j}}{M^{2}} \frac{\not p}{2}, \\
& \frac{p_{T i j}}{M^{2}} \Phi_{G G, 1}^{i j}\left(x, p_{T}^{2}\right)=\frac{1}{\pi^{2}} h_{1 T}^{\perp(B 1)}\left(x, p_{T}^{2}\right) \frac{p_{T i j} S_{T}^{i} \gamma_{5} \gamma_{T}^{j}}{M^{2}} \frac{\not P}{2}, \\
& \frac{p_{T i j}}{M^{2}} \Phi_{G G, 2}^{i j}\left(x, p_{T}^{2}\right)=\frac{1}{\pi^{2}} h_{1 T}^{\perp(B 2)}\left(x, p_{T}^{2}\right) \frac{p_{T i j} S_{T}^{i} \gamma_{5} \gamma_{T}^{j}}{M^{2}} \frac{\not P}{2}, \\
& \frac{p_{T i j}}{M^{2}} \widetilde{\Phi}_{\{\partial G\}}^{i j}\left(x, p_{T}^{2}\right)=0 .
\end{aligned}
$$

The last TMD correlator in these equations is a T-odd rank 2 TMD correlator, which is not present for a spin $1 / 2$ target. The result can also be summarized as the existence of three universal pretzelocity functions $h_{1 T}^{\perp(A)}, h_{1 T}^{\perp(B 1)}$ and $h_{1 T}^{\perp(B 2)}$ and a gauge link dependence given by

$$
h_{1 T}^{\perp[U]}\left(x, p_{T}^{2}\right)=h_{1 T}^{\perp(A)}\left(x, p_{T}^{2}\right)+C_{G G, 1}^{[U]} h_{1 T}^{\perp(B 1)}\left(x, p_{T}^{2}\right)+C_{G G, 2}^{[U]} h_{1 T}^{\perp(B 2)}\left(x, p_{T}^{2}\right) .
$$

This shows e.g. that $h_{1 T}^{\perp[\mathrm{SIDIS}]}\left(x, p_{T}^{2}\right)=h_{1 T}^{\perp[\mathrm{DY}]}\left(x, p_{T}^{2}\right)$, but that for other processes (with more complicated gauge links) other combinations of the three possible pretzelocity functions occur. In asymmetries involving $p_{T}^{\alpha \beta}$-moments of the quark TMD correlator contributions from all four correlators can appear. In particular we find for a transversely polarized spin 1/2 target three pretzelocity functions, as was already established in Eq. 18. For a spin 1/2 target our treatment is complete, since there are no higher rank TMD correlators such as $p_{T i j k} \widetilde{\Phi}_{\partial \partial \partial}^{i j k}\left(x, p_{T}^{2}\right)$. In the case of a spin $1 / 2$ target the pretzelocity TMD functions $h_{1 T}^{\perp(B 2)}$ actually was referred to as junk TMD in Ref. [8].

We want to summarize our results in this section in tabular form. We first represent the contributions in Eq. 22 in Table [I] The assignment of the TMD PDFs for an unpolarized and polarized spin $1 / 2$ target has been discussed in this section and is summarized in Tables [II and IV For the corresponding fragmentation functions the assignments are different, since gluonic pole matrix elements vanish in that case [15 17] and all functions are assigned to $\widetilde{\Phi}_{\partial \ldots \partial}$ operator structures. The assignments thus are as in Tables $\mathrm{V}$ and VI The T-odd TMD PFFs (such as the Collins function $H_{1}^{\perp}$ ) are due to the fact that the definitions of fragmentation functions involve non-plane wave states or equivalently a hadronic number operator, which are not invariant under time-reversal. There, thus, is only a single (T-even) function $H_{1 T}^{\perp}\left(z, k_{T}^{2}\right)$ appearing in the parametrization of the correlator $\Delta_{\partial \partial}^{\alpha \beta}\left(x, p_{T}^{2}\right)$. 


\begin{tabular}{|l|l|l|l|}
\hline \multicolumn{5}{|c|}{ GLUONIC POLE RANK } \\
\hline \multicolumn{1}{|c|}{0} & \multicolumn{1}{|c|}{1} & \multicolumn{1}{|c|}{2} & \multicolumn{1}{c|}{3} \\
\hline$\Phi\left(x, p_{T}^{2}\right)$ & $\pi C_{G}^{[U]} \Phi_{G}$ & $\pi^{2} C_{G G, c}^{[U]} \Phi_{G G, c}$ & $\pi^{3} C_{G G G, c}^{[U]} \Phi_{G G G, c}$ \\
\hline$\widetilde{\Phi}_{\partial}$ & $\pi C_{G}^{[U]} \widetilde{\Phi}_{\{\partial G\}}$ & $\pi^{2} C_{G G, c}^{[U]} \widetilde{\Phi}_{\{\partial G G\}, c}$ & $\cdots$ \\
\hline$\widetilde{\Phi}_{\partial \partial}$ & $\pi C_{G}^{[U]} \widetilde{\Phi}_{\{\partial \partial G\}}$ & $\ldots$ & $\cdots$ \\
\hline$\widetilde{\Phi}_{\partial \partial \partial}$ & $\ldots$ & $\ldots$ & $\cdots$ \\
\hline
\end{tabular}

TABLE II: The contributions in the TMD correlator for correlators ordered in columns according to the number of gluonic poles $(G)$ and ordered in rows according to the number of contributing partial derivatives $(\partial=D-A)$. The rank of these operators is equal to the sum of these numbers. Their twist is equal to the rank +2 .

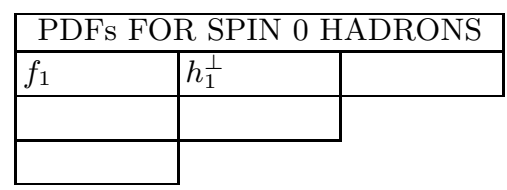

TABLE III: The assignment of TMD PDFs for a spin 0 or unpolarized target to the quark correlators as given in Table involve at most rank 1 TMD correlators. There is no T-even function corresponding to $\widetilde{\Phi}_{\partial}^{i}$.

\begin{tabular}{|l|l|l|}
\hline \multicolumn{2}{|c|}{ PFFs FOR SPIN 0 HADRONS } \\
\hline$D_{1}$ & & \\
\hline$H_{1}^{\perp}$ & & \multicolumn{1}{|c}{} \\
\hline & \multicolumn{2}{|l}{} \\
\cline { 1 - 2 } & &
\end{tabular}

TABLE V: The operator structure of quark TMD PFFs for spin 0 or unpolarized hadrons. All gluonic pole matrix elements vanish.

\begin{tabular}{|c|c|c|}
\hline \multicolumn{3}{|c|}{ PDFs FOR SPIN 1/2 HADRONS } \\
\hline$g_{1}, h_{1}$ & $f_{1 T}^{\perp}$ & $h_{1 T}^{\perp(B 1)}, h_{1 T}^{\perp(B 2)}$ \\
\hline$g_{1 T}, h_{1 L}^{\perp}$ & & \\
\hline$h_{1 T}^{\perp(A)}$ & & \\
\hline
\end{tabular}

TABLE IV: The assignment of TMD PDFs for a polarized spin $1 / 2$ target to the quark correlators as given in Table II involve at most rank 1 TMD correlators for longitudinal polarization, while they involve also rank 2 TMD correlators for a transversely polarized spin $1 / 2$ target.

\begin{tabular}{|l|l|l|}
\hline \multicolumn{2}{|l|}{ PFFs FOR SPIN 1/2 HADRONS } \\
\hline$G_{1}, H_{1}$ & & \\
\cline { 1 - 2 }$G_{1 T}, H_{1 L}^{\perp}, D_{1 T}^{\perp}$ & & \\
\cline { 1 - 1 }$H_{1 T}^{\perp}$ & \multicolumn{2}{|l}{} \\
\cline { 1 - 2 }
\end{tabular}

TABLE VI: The operator structure of quark TMD PFFs for polarized spin $1 / 2$ hadrons. Gluonic pole matrix elements vanish.

\section{B. Results for spin 1 hadrons}

Extension to higher spin targets is straightforward. We illustrate this by giving in Table VII the assignments for spin 1 tensor polarized TMD functions. These were first given in Ref. 25]. The (slightly updated) parametrization of the TMD correlator for the TMD PDFs for a tensor polarized target are given in Appendix C as well as the parametrization of the TMD PFFs in Appendix D, From these tensor polarized spin 1 contributions, the $f_{1 T T}^{[U]}\left(x, p_{T}^{2}\right)$ and $h_{1 T T}^{\perp[U]}\left(x, p_{T}^{2}\right)$ can be written as a combination of multiple universal PDFs, multiplied with process dependent gluonic pole factors,

$$
\begin{aligned}
f_{1 T T}^{[U]}\left(x, p_{T}^{2}\right) & =f_{1 T T}^{(A)}\left(x, p_{T}^{2}\right)+C_{G G, c}^{[U]} f_{1 T T}^{(B c)}\left(x, p_{T}^{2}\right), \\
h_{1 T T}^{\perp[U]}\left(x, p_{T}^{2}\right) & =C_{G}^{[U]} h_{1 T T}^{\perp(A)}\left(x, p_{T}^{2}\right)+C_{G G G, c}^{[U]} h_{1 T T}^{\perp(B c)}\left(x, p_{T}^{2}\right) .
\end{aligned}
$$

Note that the $h_{1 T T}^{\perp[U]}\left(x, p_{T}^{2}\right)$ is a rank 3 object, for which all contributing universal functions are multiplied with a process dependent prefactor. A special case is the T-odd TMD PDF $h_{1 L T}$, which is forbidden because of time-reversal invariance. Following Ref. [25], this rank 0 TMD PDF is defined as the combination $h_{1 L T}\left(x, p_{T}^{2}\right)=h_{1 L T}^{\prime}\left(x, p_{T}^{2}\right)+$ $h_{1 L T}^{\perp(1)}\left(x, p_{T}^{2}\right)$ and is shown as the wiped-out function in Table VII It shows a nice feature of our TMD functions of definite rank. In the first column only T-even TMD PDFs are allowed, in the second column only T-odd ones, etc. The first victim of the application of time-reversal invariance for leading quark TMDs, thus, is $h_{1 L T}\left(x, p_{T}^{2}\right)$, a (T-forbidden) transversely polarized quark distribution function in a tensor polarized hadron. Note that the rank 2, T-odd function $h_{1 L T}^{\perp}\left(x, p_{T}^{2}\right)$ is allowed. The only rank 0 function for a tensor polarized spin 1 target thus is $f_{1 L L}\left(x, p_{T}^{2}\right)$, introduced as the distribution $b_{1}$ in Ref. [26].

For fragmentation functions, gluonic pole contributions all vanish and only the first column survives. The parametrization of the higher rank correlators contain the T-even and T-odd TMD fragmentation functions. The 


\begin{tabular}{|c|c|c|c|}
\hline \multicolumn{4}{|c|}{ PDFs FOR TENSOR POLARIZED SPIN 1 HADRON } \\
\hline$\overline{f_{1 L L},{ }_{L K}}$ & $h_{1 L L}^{\perp}, g_{1 L T}, h_{1 T T}$ & $f_{1 T T}^{(B c)}$ & $h_{1 T T}^{\perp(B c)}$ \\
\hline$f_{1 L T}$ & $h_{1 L T}^{\perp}, g_{1 T T}$ & & \\
\hline$f_{1 T T}^{(A)}$ & $h_{1 T T}^{\perp(A)}$ & & \\
\hline
\end{tabular}

TABLE VII: The operator assignments of TMD PDFs for a tensor polarized spin 1 target require operator structures up to rank 3 . There are several different functions $f_{1 T T}\left(x, p_{T}^{2}\right)$ and $h_{1 T T}^{\perp}\left(x, p_{T}^{2}\right)$.

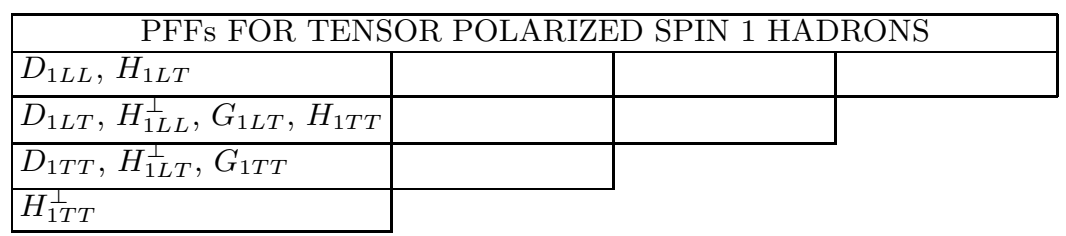

TABLE VIII: The operator structure of TMD PFFs for a tensor polarized spin 1 target requires operator structures up to rank 3 .

fragmentation functions describing fragmentation into a tensor polarized hadron are given in Table VIII.

\section{Bessel weights}

We note that the TMDs $f^{(m)}\left(x, p_{T}^{2}\right)$ of a given rank do not contain operators of definite twist. This is only true for transverse moments $f_{\ldots}^{(m)}(x)$ after $p_{T}$-integration. The TMD correlators of definite rank appearing in the parametrization in Eq. 22 only are integrated over azimuthal directions. The rank just refers to the azimuthal dependence of the correlators in the full correlator $\Phi^{[U]}\left(x, p_{T}\right)$.

Using that for a given rank $m$, there are two independent combinations $p_{T}^{i_{1} \ldots i_{m}} \propto\left|p_{T}\right|^{m} \exp \left( \pm i m \varphi_{p}\right)$, it is equivalent to consider

$$
\frac{p_{T i_{1} \ldots i_{m}}}{M^{m}} \widetilde{\Phi}_{\ldots}^{i_{1} \ldots i_{m}}\left(x, p_{T}^{2}\right) \quad \text { or } \quad \widetilde{\Phi}_{\ldots}^{(m / 2)}\left(x, p_{T}^{2}\right) e^{i m \varphi_{p}},
$$

where $\widetilde{\Phi}_{\ldots}^{(m / 2)}\left(x, p_{T}^{2}\right)=\left(-p_{T}^{2} / 2 M^{2}\right)^{m / 2} \widetilde{\Phi}_{\ldots}\left(x, p_{T}^{2}\right)$ assures the appropriate small $p_{T}$-behavior. A suitable normalization of the correlator has to assure that $\widetilde{\Phi}_{\ldots}^{(m)}\left(x, p_{T}^{2}\right)$ reproduces the collinear transverse moments upon integration,

$$
\widetilde{\Phi}_{\ldots}^{(m)}(x)=\int_{0}^{\infty} 2 \pi\left|p_{T}\right| d\left|p_{T}\right| \widetilde{\Phi}_{\cdots}^{(m)}\left(x, p_{T}^{2}\right) .
$$

Knowing the correlators in Eq. 38 to be Fourier transforms of nonlocal matrix elements in transverse space, it is natural to write the appropriately weighted TMD PDF in their parametrization as a Bessel transform,

$$
\widetilde{f}_{\ldots}^{(m / 2)}\left(x,\left|p_{T}\right|\right)=\int_{0}^{\infty} d b \sqrt{\left|p_{T}\right| b} J_{m}\left(\left|p_{T}\right| b\right) f_{\ldots}^{(m / 2)}(x, b),
$$

such that $f_{\ldots}^{(m / 2)}(x, b) \exp \left(i m \varphi_{b}\right)$ is the (two-dimensional) Fourier transform of $\widetilde{f}_{\ldots}^{(m / 2)}\left(x,\left|p_{T}\right|\right) \exp \left(i m \varphi_{p}\right)$. Bessel weightings are extensively studied in Ref. [27].

Bessel weighting may also offer a convenient way to incorporate the soft factor which usually is given in b-space [28]. This factor has been omitted from Eq. 19. Our decomposition in Eq. 22, however, can always be written down, but the $\Phi_{\ldots}\left(x, p_{T}^{2}\right)$ will be modified by the inclusion of the soft factor.

\section{CONCLUSIONS}

In Eq. 22 we have presented a parametrization for TMD quark correlators that distinguishes different azimuthal dependences. For this we write down an expansion in terms of irreducible tensors in the transverse momentum multiplied with correlators depending on $x$ and $p_{T}^{2}$. These correlators contain tensors describing the polarization of the target and TMD functions depending on $x$ and $p_{T}^{2}$. The rank of the irreducible tensors in transverse momentum 
space also defines the rank of the correlators and TMD functions multiplying this tensor. The field theoretical expression for the quark correlator basically has two quark fields connected by a gauge link. The operator structure of the TMD correlators of a definite rank contain Dirac gamma matrices, derivatives or gauge fields with transverse indices in color gauge-invariant combinations and a definite rank. They are structured in a similar way as higher twist operators in the collinear case. Each independent operator combination defines a particular TMD function. For leading twist operators the relevant 'transverse' operators are either gluonic pole $(G)$ or partial derivative $(\partial=D-A)$ or combinations thereof. A special feature of these operator combinations of definite rank is that they are either Teven or T-odd after extraction of a gluonic pole factor without having to perform weighting and integration over transverse momentum.

Using the parametrization of TMD correlators in Eq. 22, one finds that rank 0 and rank 1 contributions are similar to previously used definitions for T-even and T-odd contributions, such as e.g. obtained by combining 'opposite' gauge links in Eq. 8. Just as the collinear transverse moments, these TMD functions are universal functions, multiplied with a process dependent prefactor, rather than nonuniversal gauge link dependent functions. By explicitly and systematically looking at all contributing functions, one finds for an unpolarized target two TMD quark correlators, the first being of rank 0 , containing the TMD PDF $f_{1}\left(x, p_{T}^{2}\right)$. Looking at the operator structure, it is interpreted as the momentum distribution of quarks. The second unpolarized TMD correlator is a T-odd gluonic pole matrix element of rank 1. It contains the Boer-Mulders TMD PDF $h_{1}^{\perp}\left(x, p_{T}^{2}\right)$.

For a polarized spin 1/2 target there are an additional eight TMD correlators, containing rank 0 , rank 1 and rank 2 contributions. The two rank 0 correlators contain the TMD PDFs that are interpreted as the well-known polarized spin distribution functions in longitudinally or transversely polarized targets. For a longitudinally polarized target, there is a T-even rank 1 TMD correlator $\widetilde{\Phi}_{\partial}\left(x, p_{T}^{2}\right)$ containing the worm gear function $h_{1 L}^{\perp}\left(x, p_{T}^{2}\right)$. For a transversely polarized target, there exist one T-even TMD correlator $\widetilde{\Phi}_{\partial}\left(x, p_{T}^{2}\right)$ and a T-odd rank 1 TMD correlator $\Phi_{G}\left(x, p_{T}^{2}\right)$ containing the worm gear function $g_{1 T}\left(x, p_{T}^{2}\right)$ and the Sivers function $f_{1 T}^{\perp}\left(x, p_{T}^{2}\right)$, respectively. The three TMD correlators of rank 2 appear in the T-even correlator $\widetilde{\Phi}_{\partial \partial}\left(x, p_{T}^{2}\right)$ and the two T-even double gluonic pole correlators $\Phi_{G G, c}\left(x, p_{T}^{2}\right)$, giving rise to the pretzelocity functions $h_{1 T}^{\perp(A)}\left(x, p_{T}^{2}\right), h_{1 T}^{\perp(B 1)}\left(x, p_{T}^{2}\right)$ and $h_{1 T}^{\perp(B 2)}\left(x, p_{T}^{2}\right)$. These functions in general both show up in particular azimuthal asymmetries but with gauge link dependent prefactors, where the gauge link in turn depends on the process. As for the functions themselves and in particular their interpretation, the function $h_{1 T}^{\perp(A)}$ is related to the quark structure of a nucleon, while the functions $h_{1 T}^{\perp(B c)}$ are the ones involving quark-gluon correlations.

For a spin 1 target one finds apart from the above mentioned TMD correlators additional correlators because one also has the possibility of tensor polarization. The full list of TMDs has been given in Table VII including rank 3 contributions coming with process dependent prefactors. Rank 3 contributions like this are specific for targets with spin 1 or higher.

The procedure for defining universal TMD correlators of definite rank can be extended to gluon TMDs and to higher twist situations. The extension to gluon TMDs will be presented in a forthcoming publication. The situation for higher twist TMDs is complicated by the fact that the lowest twist operators that contribute to the TMDs not only contain two quark fields or two gluon fields, but they also contain additional (gluon) operators with transverse directions, $D_{T}^{\alpha}$ and $F_{T}^{n \alpha}$, no longer in the combination $\partial_{T}^{\alpha}$.

As a final advantage of the universal TMD correlators we mention that, although their nonlocal operator structure is of the form in Eq. 19 with a particular gauge link $U$, the TMD correlators of definite rank have definite T-behavior (even or odd) and are independent of the gauge link. The $U$-dependence is in the gluonic pole factors and the color structure of the operator combination. Thus one can study the universal TMD correlators, for instance in lattice calculations, by using just the sum and difference of the simplest $U^{[+]}$and $U^{[-]}$staple links.

\section{Acknowledgements}

This research is part of the research program of the "Stichting voor Fundamenteel Onderzoek der Materie (FOM)", which is financially supported by the "Nederlandse Organisatie voor Wetenschappelijk Onderzoek (NWO)". MGAB also acknowledges support of the FP7 EU-programme HadronPhysics3 (contract no 283286). AM thanks the Alexander von Humboldt Fellowship for Experienced Researchers, Germany, for support. AM also acknowledges support from the FOM Programme "Theoretical Physics in the LHC Era", for visiting VU University and Nikhef, Amsterdam, where this work was initiated. We would like to acknowledge discussions with several participants of the QCD Evolution Workshop at Jefferson Lab (14-17 May 2012). All figures were made using JaxoDraw [29, 30]. 


\section{Appendix A: Double weighted collinear matrix elements}

Double $p_{T}$-weighting is worked out in the same way as the single weighting. In evaluating the weighting one can actually choose the derivatives to work on $\xi$ or 0 or mix these, but that does not matter for the final answer. Since we just are interested in the general structure, we work with the multi-parton element with all gluons on the left side of the cut. For the gauge link $U_{[0, \xi]}^{[+]}$we note that (writing only the relevant pieces of the matrix elements) [31]

$$
i \partial_{T}^{\alpha} U_{[0, \xi]}^{[+]}=U_{[0, \infty]}^{[n]} i \partial_{T}^{\alpha} U_{\left[0_{T}, \xi_{T}\right]}^{T} U_{[\infty, \xi]}^{[n]}=U_{[0, \infty]}^{[n]} U_{\left[0_{T}, \xi_{T}\right]}^{T} i D_{T}^{\alpha} U_{[\infty, \xi]}^{[n]},
$$

which is then further evaluated using

$$
i D_{T}^{\alpha} U_{[\infty, \xi]}^{[n]} \ldots \psi(\xi)=U_{[\infty, \xi]}^{[n]}\left(i D_{T}^{\alpha}(\xi)-A_{T}^{\alpha}(\xi)+\pi \widetilde{G}^{n \alpha}(\xi)\right) \ldots \psi(\xi) .
$$

The $A_{T}^{\alpha}(\xi)$ and $\pi \widetilde{G}^{n \alpha}(\xi)$ are defined as

$$
\begin{aligned}
& A_{T}^{\alpha}(\xi)=\frac{1}{2} \int_{-\infty}^{\infty} d \eta \cdot P \epsilon(\xi \cdot P-\eta \cdot P) U_{[\xi, \eta]}^{[n]} G^{n \alpha}(\eta) U_{[\eta, \xi]}^{[n]}, \\
& \pi \widetilde{G}^{n \alpha}(\xi)=\frac{1}{2} \int_{-\infty}^{\infty} d \eta \cdot P U_{[\xi, \eta]}^{[n]} G^{n \alpha}(\eta) U_{[\eta, \xi]}^{[n]},
\end{aligned}
$$

with $\epsilon(\zeta)$ being the sign function taking the values $1,-1$ and 0 . Note that $\widetilde{G}^{n \alpha}(\xi)=\widetilde{G}^{n \alpha}\left(\xi \cdot P, \xi_{T}\right)$ does not depend on $\xi \cdot n$. The above described method for calculating the structure of the matrix elements for a single transverse weighting can be extended to higher transverse weightings by repeated application of Eq. A2. For example, for the $U^{[+]}$gauge link this implies

$$
\begin{aligned}
i D_{T}^{\alpha} i D_{T}^{\beta} U_{[\infty, \xi]}^{[n]} \ldots \psi(\xi) & \\
= & U_{[\infty, \xi]}^{[n]}\left(\left(i D_{T}^{\alpha}(\xi)-A_{T}^{\alpha}(\xi)\right)\left(i D_{T}^{\beta}(\xi)-A_{T}^{\beta}(\xi)\right)\right) \ldots \psi(\xi)+U_{[\infty, \xi]}^{[n]}\left(\pi \widetilde{G}^{n \alpha}(\xi) \pi \widetilde{G}^{n \beta}(\xi)\right) \ldots \psi(\xi) \\
& \quad+U_{[\infty, \xi]}^{[n]}\left(\pi \widetilde{G}^{n \alpha}(\xi)\left(i D_{T}^{\beta}(\xi)-A_{T}^{\beta}(\xi)\right)\right) \ldots \psi(\xi)+U_{[\infty, \xi]}^{[n]}\left(\left(i D_{T}^{\alpha}(\xi)-A_{T}^{\alpha}(\xi)\right) \pi \widetilde{G}^{n \beta}(\xi)\right) \ldots \psi(\xi) .
\end{aligned}
$$

After integration over $p_{T}$ the resulting correlators can be rewritten in terms of color gauge-invariant multi-parton correlators as was done for the single weighting. In this case one needs the correlators of the form

$$
\begin{aligned}
\Phi_{O_{1} O_{2}}^{\alpha \beta}\left(x-x_{1}-x_{2}, x_{1}, x_{2} \mid x\right)= & \int \frac{d \xi \cdot P}{2 \pi} \frac{d \eta \cdot P}{2 \pi} \frac{d \eta^{\prime} \cdot P}{2 \pi} e^{i x_{2}\left(\eta^{\prime} \cdot P\right)} e^{i x_{1}(\eta \cdot P)} e^{i\left(x-x_{1}-x_{2}\right)(\xi \cdot P)} \\
& \left.\times\left\langle P, S\left|\bar{\psi}(0) U_{\left[0, \eta^{\prime}\right]}^{[n]} O_{1 T}^{\alpha}\left(\eta^{\prime}\right) U_{\left[\eta^{\prime}, \eta\right]}^{[n]} O_{2 T}^{\beta}(\eta) U_{[\eta, \xi]}^{[n]} \psi(\xi)\right| P, S\right\rangle\right\rfloor_{L C},
\end{aligned}
$$

with $O_{1 T}^{\alpha}$ and $O_{2 T}^{\alpha}$ hermitian operators like $i D_{T}^{\alpha}$ and/or $F_{T}^{n \alpha}$. In general more than one color structure is possible. As an example, for the operator combination $\bar{\psi} F F \psi$, one can have the two distinct color configurations

$$
\begin{array}{ll}
c=1: & \operatorname{Tr}_{c}[F F \psi \bar{\psi}]=\bar{\psi} F F \psi=\bar{\psi}^{r} F^{r s^{\prime}} F^{s^{\prime} s} \psi^{s}, \\
c=2: & \operatorname{Tr}_{c}[F F] \operatorname{Tr}_{c}[\psi \bar{\psi}]=\bar{\psi} \psi \operatorname{Tr}_{c}[F F]=\bar{\psi}^{r} \psi^{r} F^{s s^{\prime}} F^{s^{\prime} s} .
\end{array}
$$

An example of a correlator one needs is

$$
\begin{aligned}
\Phi_{D D}^{\alpha \beta}(x) & \left.=\int \frac{d \xi \cdot P}{2 \pi} e^{i x(\xi \cdot P)}\left\langle P, S\left|\bar{\psi}(0) U_{[0, \xi]}^{[n]} i D_{T}^{\alpha}(\xi) i D_{T}^{\beta}(\xi) \psi(\xi)\right| P, S\right\rangle\right\rfloor_{L C} \\
& =\int d x_{1} d x_{2} \Phi_{D D}^{\alpha \beta}\left(x-x_{1}-x_{2}, x_{1}, x_{2} \mid x\right) .
\end{aligned}
$$


Other correlators involving $A_{T}^{\alpha}(\xi)$ or $\pi \widetilde{G}_{T}^{n \alpha}(\xi)$, given in Eqs A3 and A4, are given by

$$
\begin{aligned}
\Phi_{A A}^{\alpha \beta}(x) & =\int d x_{1} \mathrm{PV} \frac{i}{x_{1}} \int d x_{2} \mathrm{PV} \frac{i}{x_{2}} \Phi_{F F}^{\alpha \beta}\left(x-x_{1}-x_{2}, x_{1}, x_{2} \mid x\right), \\
\Phi_{A D}^{\alpha \beta}(x) & =\int d x_{1} d x_{2} \operatorname{PV} \frac{i}{x_{1}} \Phi_{F D}^{\alpha \beta}\left(x-x_{1}-x_{2}, x_{1}, x_{2} \mid x\right), \\
\Phi_{D A}^{\alpha \beta}(x) & =\int d x_{1} d x_{2} \operatorname{PV} \frac{i}{x_{2}} \Phi_{D F}^{\alpha \beta}\left(x-x_{1}-x_{2}, x_{1}, x_{2} \mid x\right), \\
\Phi_{G D}^{\alpha \beta}(x) & =\int d x_{2} \Phi_{F D}^{\alpha \beta}\left(x-x_{2}, 0, x_{2} \mid x\right), \\
\Phi_{D G}^{\alpha \beta}(x) & =\int d x_{1} \Phi_{D F}^{\alpha \beta}\left(x-x_{1}, x_{1}, 0 \mid x\right), \\
\Phi_{G A}^{\alpha \beta}(x) & =\int d x_{2} \operatorname{PV} \frac{i}{x_{2}} \Phi_{F F}^{\alpha \beta}\left(x-x_{2}, 0, x_{2} \mid x\right), \\
\Phi_{A G}^{\alpha \beta}(x) & =\int d x_{1} \operatorname{PV} \frac{i}{x_{1}} \Phi_{F F}^{\alpha \beta}\left(x-x_{1}, x_{1}, 0 \mid x\right), \\
\Phi_{G G, c}^{\alpha \beta}(x) & =\Phi_{F F, c}^{\alpha \beta}(x, 0,0 \mid x) .
\end{aligned}
$$

Using for the correlators, just as for the single weighted case, the notation $\widetilde{\Phi}_{\partial \ldots}$ for the correlators with covariant derivative minus a correlator with a principal value integration $\left(i D_{T}-A_{T}\right)$, implies

$$
\begin{aligned}
& \widetilde{\Phi}_{\partial \partial}^{\alpha \beta}(x)=\Phi_{D D}^{\alpha \beta}(x)-\Phi_{D A}^{\alpha \beta}(x)-\Phi_{A D}^{\alpha \beta}(x)+\Phi_{A A}^{\alpha \beta}(x), \\
& \widetilde{\Phi}_{\partial G}^{\alpha \beta}(x)=\Phi_{D G}^{\alpha \beta}(x)-\Phi_{A G}^{\alpha \beta}(x), \\
& \widetilde{\Phi}_{G \partial}^{\alpha \beta}(x)=\Phi_{G D}^{\alpha \beta}(x)-\Phi_{G A}^{\alpha \beta}(x) .
\end{aligned}
$$

The second transverse moment in terms of the collinear functions then is (symmetrizing in $\alpha$ and $\beta$ )

$$
\int d^{2} p_{T} p_{T}^{\{\alpha} p_{T}^{\beta\}} \Phi^{[+]}\left(x, p_{T}\right)=\widetilde{\Phi}_{\partial \partial}^{\{\alpha \beta\}}(x)+\pi \widetilde{\Phi}_{\partial G}^{\{\alpha \beta\}}(x)+\pi \widetilde{\Phi}_{G \partial}^{\{\alpha \beta\}}(x)+\pi^{2} \Phi_{G G, 1}^{\{\alpha \beta\}}(x),
$$

which is the result given in Eq. 17 with $C_{G}^{[+]}=1, C_{G G, 1}^{[+]}=1$ and $C_{G G, 2}^{[+]}=0$. For other gauge link structures, similar calculations can be performed.

\section{Appendix B: Double weighted TMD matrix elements}

The leading relevant TMD operator structure for our considerations referred to in Eq. 19 is bilocal,

$$
\Phi^{[U]}\left(x, p_{T} ; n\right)=\left.\int \frac{d \xi \cdot P d^{2} \xi_{T}}{(2 \pi)^{3}} e^{i p \cdot \xi}\left\langle P\left|\bar{\psi}(0) U_{[0, \xi]} O(\xi)\right| P\right\rangle\right|_{\xi \cdot n=0} .
$$

The nonlocality, however, involves a transverse separation, hence the gauge link $U_{[0, \xi]}$ in general can be complicated. For the two cases $c=1,2$ one now finds for the gauge link $U_{[0, \xi]}^{[(\square)+]}$ the nonlocal structures

$$
\begin{array}{ll}
c=1: & \operatorname{Tr}_{c}\left[U_{[0, \xi]}^{[(\square)+]} \widetilde{G}(\xi) \widetilde{G}(\xi) \psi(\xi) \bar{\psi}(0)\right]=\bar{\psi}(0) U_{[0, \xi]}^{[(\square)+]} \widetilde{G}(\xi) \widetilde{G}(\xi) \psi(\xi), \\
c=2: & \operatorname{Tr}_{c}\left[U_{[0, \xi]}^{[\square]} \widetilde{G}(\xi) \widetilde{G}(\xi)\right] \operatorname{Tr}_{c}\left[U_{[0, \xi]}^{[+]} \psi(\xi) \bar{\psi}(0)\right]=\bar{\psi}(0) U_{[0, \xi]}^{[+]} \psi(\xi) \operatorname{Tr}_{c}\left[U_{[0, \xi]}^{[+]} \widetilde{G}(\xi) \widetilde{G}(\xi) U_{[\xi, 0]}^{\left.[-]^{\dagger}\right]} .\right.
\end{array}
$$

\section{Appendix C: Parametrization of the spin 1 distribution correlator}

The parametrization of a distribution correlator for a spin 1 hadron was first given in Ref. [25] and is given by

$$
\Phi\left(x, p_{T}\right)=\Phi_{U}\left(x, p_{T}\right)+\Phi_{L}\left(x, p_{T}\right)+\Phi_{T}\left(x, p_{T}\right)+\Phi_{L L}\left(x, p_{T}\right)+\Phi_{L T}\left(x, p_{T}\right)+\Phi_{T T}\left(x, p_{T}\right),
$$


where the contributions $\Phi_{U}\left(x, p_{T}\right), \Phi_{L}\left(x, p_{T}\right)$ and $\Phi_{T}\left(x, p_{T}\right)$ are parametrized in the same way as those contributions in the correlators that describe an unpolarized, longitudinally or transversely polarized spin $1 / 2$ particle, given in Eq. 3 using the notation of Ref. 24]. We update the parametrization for the remaining correlators contributing for spin 1 particles, using the same TMD PDFs as in Ref. [25]. This leads for the leading twist TMDs to

$$
\begin{aligned}
& \Phi_{L L}\left(x, p_{T}\right)=\left\{f_{1 L L}\left(x, p_{T}^{2}\right) S_{L L}+i h_{1 L L}^{\perp}\left(x, p_{T}^{2}\right) S_{L L} \frac{\not p_{T}}{M}\right\} \frac{\not P}{2}, \\
& \Phi_{L T}\left(x, p_{T}\right)=\left\{-f_{1 L T}\left(x, p_{T}^{2}\right) \frac{p_{T} \cdot S_{L T}}{M}+g_{1 L T}\left(x, p_{T}^{2}\right) \epsilon_{T}^{\mu \nu} S_{L T} \mu \frac{p_{T \nu}}{M} \gamma_{5}\right. \\
& \left.+h_{1 L T}^{\prime}\left(x, p_{T}^{2}\right) \gamma_{5} \gamma_{\nu} \epsilon_{T}^{\nu \rho} S_{L T \rho}-i h_{1 L T}^{\perp}\left(x, p_{T}^{2}\right) \frac{p_{T} \cdot S_{L T}}{M} \frac{\not p_{T}}{M}\right\} \frac{\not p}{2}, \\
& \Phi_{T T}\left(x, p_{T}\right)=\left\{f_{1 T T}\left(x, p_{T}^{2}\right) \frac{p_{T \alpha \beta} S_{T T}^{\alpha \beta}}{M^{2}}-g_{1 T T}\left(x, p_{T}^{2}\right) \epsilon_{T}^{\mu \nu} S_{T T \nu \rho} \frac{p_{T}^{\rho} p_{T \mu}}{M^{2}} \gamma_{5}\right. \\
& \left.-h_{1 T T}^{\prime}\left(x, p_{T}^{2}\right) \gamma_{5} \gamma_{\nu} \epsilon_{T}^{\nu \rho} S_{T T} \rho \sigma \frac{p_{T}^{\sigma}}{M}+i h_{1 T T}^{\perp}\left(x, p_{T}^{2}\right) \frac{p_{T \alpha \beta} S_{T T}^{\alpha \beta}}{M^{2}} \frac{\not p_{T}}{M}\right\} \frac{\not P}{2} .
\end{aligned}
$$

We note that all polarized quark distributions ( $g$ and $h$ functions) in a tensor polarized target, $h_{1 L L}^{\perp}, g_{1 L T}, h_{1 L T}^{\prime}$, $h_{1 L T}^{\perp}, g_{1 T T}, h_{1 T T}^{\prime}$ and $h_{1 T T}^{\perp}$, are T-odd. Just as in Ref. [25], the integrated case is given by

$$
\begin{aligned}
\Phi_{L L}(x) & =f_{1 L L}(x) S_{L L} \frac{\not p}{2}, \\
\Phi_{L T}(x) & =h_{1 L T}(x) \gamma_{\nu} \epsilon_{T}^{\nu \rho} S_{L T} \rho \frac{\not P}{2}, \\
\Phi_{T T}(x) & =0
\end{aligned}
$$

where

$$
h_{1 L T}\left(x, p_{T}^{2}\right)=h_{1 L T}^{\prime}\left(x, p_{T}^{2}\right)+h_{1 L T}^{\perp(1)}\left(x, p_{T}^{2}\right) .
$$

For identifying the proper rank 1 TMD, it is also useful to define

$$
h_{1 T T}\left(x, p_{T}^{2}\right)=h_{1 T T}^{\prime}\left(x, p_{T}^{2}\right)+h_{1 T T}^{\perp(1)}\left(x, p_{T}^{2}\right) .
$$

\section{Appendix D: Parametrization of the fragmentation correlator}

The parametrization of the fragmentation correlator for a spin 1 particle, also first used in Ref. [25], is similar in structure to the parametrization of the distribution correlator and is given by

$$
\Delta\left(z, k_{T}\right)=\Delta_{U}\left(z, k_{T}\right)+\Delta_{L}\left(z, k_{T}\right)+\Delta_{T}\left(z, k_{T}\right)+\Delta_{L L}\left(z, k_{T}\right)+\Delta_{L T}\left(z, k_{T}\right)+\Delta_{T T}\left(z, k_{T}\right),
$$

where the $\Delta_{U}\left(z, k_{T}\right), \Delta_{L}\left(z, k_{T}\right)$ and $\Delta_{T}\left(z, k_{T}\right)$ are the correlators that describe fragmentation into an unpolarized, longitudinally and transversely polarized spin $1 / 2$ particle. For spin 0 only $\Delta_{U}\left(z, k_{T}\right)$ is relevant. The correlators in Eq. D1] are with the notation of Ref. [24] at leading twist given by

$$
\begin{aligned}
\Delta_{U}\left(z, k_{T}\right)= & \left\{D_{1}\left(z, k_{T}^{2}\right)+i H_{1}^{\perp}\left(z, k_{T}^{2}\right) \frac{\not k_{T}}{M_{h}}\right\} \frac{\not K}{2}, \\
\Delta_{L}\left(z, k_{T}\right)= & \left\{G_{1 L}\left(z, k_{T}^{2}\right) S_{h_{L}} \gamma_{5}+H_{1 L}^{\perp}\left(z, k_{T}^{2}\right) S_{h_{L}} \frac{\gamma_{5} k_{T}}{M_{h}}\right\} \frac{\not K}{2}, \\
\Delta_{T}\left(z, k_{T}\right)= & \left\{-G_{1 T}\left(z, k_{T}^{2}\right) \frac{k_{T} \cdot S_{h_{T}}}{M_{h}} \gamma_{5}+H_{1 T}\left(z, k_{T}^{2}\right) \gamma_{5} \$_{h T}\right. \\
& \left.-H_{1 T}^{\perp}\left(z, k_{T}^{2}\right) \frac{k_{T} \cdot S_{h_{T}}}{M_{h}} \frac{\gamma_{5}}{M_{h}}+D_{1 T}^{\perp}\left(z, k_{T}^{2}\right) \frac{\epsilon_{T}^{\rho \sigma} k_{T \rho} S_{h T \sigma}}{M_{h}}\right\} \frac{\not K}{2},
\end{aligned}
$$




$$
\begin{aligned}
& \Delta_{L L}\left(z, k_{T}\right)=\left\{D_{1 L L}\left(z, k_{T}^{2}\right) S_{h_{L L}}+i H_{1 L L}^{\perp}\left(z, k_{T}^{2}\right) S_{h_{L L} L} \frac{k_{T}}{M_{h}}\right\} \frac{\not K}{2}, \\
& \Delta_{L T}\left(z, k_{T}\right)=\left\{-D_{1 L T}\left(z, k_{T}^{2}\right) \frac{k_{T} \cdot S_{h_{L T}}}{M_{h}}-G_{1 L T}\left(z, k_{T}^{2}\right) \epsilon_{T}^{\mu \nu} S_{h L T} \frac{k_{T \nu}}{M_{h}} \gamma_{5}\right. \\
& \left.-H_{1 L T}^{\prime}\left(z, k_{T}^{2}\right) \gamma_{5} \gamma_{\nu} \epsilon_{T}^{\nu \rho} S_{h_{L T} \rho}-i H_{1 L T}^{\perp}\left(z, k_{T}^{2}\right) \frac{k_{T} \cdot S_{h_{L T}}}{M_{h}} \frac{\not k_{T}}{M_{h}}\right\} \frac{\not K}{2}, \\
& \Delta_{T T}\left(z, k_{T}\right)=\left\{D_{1 T T}\left(z, k_{T}^{2}\right) \frac{k_{T \alpha \beta} S_{h T T}^{\alpha \beta}}{M_{h}^{2}}+G_{1 T T}\left(z, k_{T}^{2}\right) \epsilon_{T}^{\mu \nu} S_{h T T \nu \rho} \frac{k_{T}^{\rho} k_{T \mu}}{M_{h}^{2}} \gamma_{5}\right. \\
& \left.+H_{1 T T}^{\prime}\left(z, k_{T}^{2}\right) \gamma_{5} \gamma_{\nu} \epsilon_{T}^{\nu \rho} S_{h T T \rho \sigma} \frac{k_{T}^{\sigma}}{M_{h}}+i H_{1 T T}^{\perp}\left(z, k_{T}^{2}\right) \frac{k_{T \alpha \beta} S_{h T T}^{\alpha \beta}}{M_{h}^{2}} \frac{k_{T}}{M_{h}}\right\} \frac{\not K}{2},
\end{aligned}
$$

where $D_{1 T}^{\perp}, H_{1}^{\perp}, H_{1 L L}^{\perp}, G_{1 L T}, H_{1 L T}^{\prime}, H_{1 L T}^{\perp}, G_{1 T T}, H_{1 T T}^{\prime}$ and $H_{1 T T}^{\perp}$ are T-odd. The relative sign difference between certain corresponding TMD PDF and TMD PFF contributions comes from the definition $\epsilon_{T}^{\alpha \beta}=\epsilon^{\alpha \beta \rho \sigma} n_{+\rho} n_{-\sigma}$, where interchanging $n_{+}$and $n_{-}$gives a relative minus sign [24]. The TMD PFFs $G_{1}\left(z, k_{T}^{2}\right), H_{1}\left(z, k_{T}^{2}\right), H_{1 L T}\left(z, k_{T}^{2}\right)$ and $H_{1 T T}\left(z, k_{T}^{2}\right)$ are defined in the same way as their TMD PDF counterparts, whereas integrated TMD PFFs are defined as

$$
D_{\ldots}(z)=z^{2} \int d^{2} k_{T} D \ldots\left(z, k_{T}^{2}\right)
$$

[1] J.C. Collins, Phys. Lett. B 536, 43 (2002), hep-ph/0204004.

[2] D. Boer, P.J. Mulders and F. Pijlman, Nucl. Phys. B 667, 201 (2003), hep-ph/0303034.

[3] A.V. Belitsky, X. Ji and F. Yuan, Nucl. Phys. B 656, 165 (2003), hep-ph/0208038.

[4] C.J. Bomhof, P.J. Mulders and F. Pijlman, Phys. Lett. B 596, 277 (2004), hep-ph/0406099.

[5] S.J. Brodsky, D.S. Hwang and I. Schmidt, Phys. Lett. B 530, 99 (2002), hep-ph/0201296.

[6] S.J. Brodsky, D.S. Hwang and I. Schmidt, Nucl. Phys. B 642, 344 (2002), hep-ph/0206259

[7] A. Bacchetta, C.J. Bomhof, P.J. Mulders and F. Pijlman, Phys. Rev. D 72, 034030 (2005), hep-ph/0505268

[8] C.J. Bomhof and P.J. Mulders, Nucl. Phys. B 795, 409 (2008), arXiv:0709.1390 [hep-ph].

[9] A.V. Efremov and O.V. Teryaev, Sov. J. Nucl. Phys. 36, 140 (1982).

[10] A.V. Efremov and O.V. Teryaev, Phys. Lett. B 150, 383 (1985).

[11] J-W. Qiu and G.F. Sterman, Phys. Rev. Lett. 67, 2264 (1991).

[12] J-W. Qiu and G.F. Sterman, Nucl. Phys. B 378, 52 (1992).

[13] J-W. Qiu and G.F. Sterman, Phys. Rev. D 59, 014004 (1998), hep-ph/9806356

[14] Y. Kanazawa and Y. Koike, Phys. Lett. B 478, 121 (2000), hep-ph/0001021.

[15] L.P. Gamberg, A. Mukherjee and P.J. Mulders, Phys. Rev. D 83, 071503 (2011), arXiv:1010.4556 [hep-ph].

[16] S. Meissner and A. Metz, Phys. Rev. Lett. 102, 172003 (2009), arXiv:0812.3783 [hep-ph].

[17] L.P. Gamberg, A. Mukherjee and P.J. Mulders, Phys. Rev. D 77, 114026 (2008), arXiv:0803.2632 [hep-ph].

[18] J.C. Collins and A. Metz, Phys. Rev. Lett. 93, 252001 (2004), hep-ph/0408249

[19] A. Metz, Phys. Lett. B 549, 139 (2002), hep-ph/0209054.

[20] P.J. Mulders and R.D. Tangerman, Nucl. Phys. B 461, 197 (1996), hep-ph/9510301 [Erratum-ibid. B 484, 538 (1997)].

[21] H. Avakian, A.V. Efremov, P. Schweitzer and F. Yuan, Phys. Rev. D 78, 114024 (2008), arXiv:0805.3355 [hep-ph].

[22] P.J. Mulders and R.D. Tangerman, Phys. Rev. D 51, 3357 (1995), hep-ph/9403227

[23] J. Zhu and B-Q. Ma, Phys.Rev. D 82114022 (2010), arXiv:1103.4201 [hep-ph].

[24] A. Bacchetta, M. Diehl, K. Goeke, A. Metz, P.J. Mulders and M. Schlegel, JHEP 0702, 093 (2007), hep-ph/0611265

[25] A. Bacchetta and P.J. Mulders, Phys. Rev. D 62, 114004 (2000), hep-ph/0007120.

[26] P. Hoodbhoy, R.L. Jaffe and A. Manohar, Nucl. Phys. B 312, 571 (1989).

[27] D. Boer, L. Gamberg, B. Musch and A. Prokudin, JHEP 1110, 021 (2011), arXiv:1107.5294 [hep-ph].

[28] J.C. Collins, Foundations of perturbative QCD, Cambridge University Press, Cambridge U.K. (2011).

[29] D. Binosi and L. Theussl, Comput. Phys. Commun. 161, 76 (2004), hep-ph/0309015.

[30] D. Binosi, J. Collins, C. Kaufhold and L. Theussl, Comput. Phys. Commun. 180, 1709 (2009), arXiv:0811.4113 [hep-ph].

[31] M.G.A. Buffing and P.J. Mulders, JHEP 1107, 065 (2011), arXiv:1105.4804 [hep-ph]. 